\title{
EFECTO DEL ÁCIDO SALICÍLICO EN EL CONTROL DE LA SEPTORIOSIS Y SU EFECTO DE RENDIMIENTO EN DOS CULTIVARES DE TRIGO
}

\author{
EFFECT OF SALICYLIC ACID IN THE CONTROL OF SEPTORIA \\ AND YIELD COMPONENTS IN TWO WHEAT CULTIVARS
}

\section{${ }^{1}$ Efren Venancio Ramos Cabrera, 2 Zuly Yuliana Delgado Espinosa, ${ }^{3}$ Oscar Daniel Jiménez}

1 PhD. Microbiología, Investigador grupo de investigación cadenas de valor. Corporación Universitaria Comfacauca-Unicomfacauca Popayán, Colombia

2 PhD. en ciencias química, Investigador grupo de investigación cadenas de valor. Corporación Universitaria Comfacauca-Unicomfacauc. Popayán, Colombia.

${ }^{3}$ Ingeniero agrónomo. Docente de la cátedra de fisiología vegetal de la facultad de ciencias agraria de forestales-universidad nacional de la plata. La Plata, Argentina

\footnotetext{
${ }^{1}$ eramos@unicomfacauca.edu.co, ${ }^{2}$ zdelgado@unicomfacauca.edu.co, ${ }^{3}$ dcbiologicas@agro.unlp.edu.ar
}

\section{RESUMEN}

Contextualización: el incremento de la producción de trigo ha provocado que las enfermedades patogénicas aparezcan con más frecuencia por la implementación del monocultivo y malas prácticas agronómicas. La Septoriosis - Mancha de la hoja del trigo ocasionada por Zymoseptoria tritici provoca una disminución en el área fotosintética ocasionando pérdidas del rendimiento del grano que oscilan entre 17 y $50 \%$, dependiendo del estado fenológico en el que ocurre la infección.

Vacío de conocimiento: el método más frecuentemente para el control de la enfermedad se realiza mediante dos sistemas: la utilización de variedades resistentes a la enfermedad o la aplicación de productos de síntesis química que pueden ocasionar resistencia en la población del hongo y contaminación en los suelos trigueros. Por esto, se buscan nuevas alternativas de manejo, como la activación de la resistencia sistémica inducida mediante la aplicación de ácido salicílico, que conlleva a cambios físicos, químicos y moleculares como lignificación o inducción de proteínas relacionadas con la patogénesis, que se encargan de limitar o eliminar el ingreso de las hifas de los hongos patogénicos, siendo una alternativa sustentable. 
Propósito del estudio: el propósito del presente trabajo fue la evaluación del efecto del ácido salicílico sobre el desarrollo de los síntomas de la mancha de hoja del trigo ocasionada por Zymoseptoria tritici, así como establecer los cambios sobre los componentes de rendimiento en los cultivares Biointa 3004 y Buck sy 200.

Metodología: el estudio se realizó en la estación experimental de Julio Hirschhorn, de la Universidad Nacional de La Plata (UNLP). Se utilizaron los cultivares Biointa 3004 y Buck sy 200. El diseño experimental fue bloques completos al azar de tres repeticiones, constituido por los siguientes tratamientos: control, inoculado con Zymoseptoria tritici, tratado con ácido salicílico y tratado con ácido salicílico e inoculado con Zymoseptoria tritici, los ensayos fueron desarrollados en la temporada inviernoprimavera del año 2012 y 2013 y se determinó porcentaje de picnidios, porcentaje área necrosada, número de espiguillas vacías, número de granos de la espiga y peso de 1000 granos.

Resultados y conclusiones: la cobertura de picnidios disminuyó un 45\%; el área necrosada un 40\%; además el número de espiguillas vacías disminuyó un 4\% e incrementó el número de granos por espiga (5\%) y peso de 1000 granos (2\%). Se demuestra que el ácido salicílico controla en gran medida los efectos negativos de la enfermedad y estimula los componentes de rendimiento mejorando la productividad de los cultivares evaluados.

Palabras clave: Zymoseptoria tritici, Triticum aestivum, inducción de resistencia, mancha de la hoja del trigo.

\section{ABSTRACT}

Contextualization: The increase in wheat production has caused pathogenic diseases to appear more frequently due to the implementation of monoculture. The Septoriosis or stain of the wheat leaf caused by Zymoseptoria tritici causes a decrease in the photosynthetic area causing losses in grain yield ranging between $17 \%$ and $50 \%$, depending on the phenological state in which the infection occurs.

Knowledge gap: The most frequent method for controlling the disease is the application of chemical synthesis products, which has generated resistance in the population of the fungus and contamination of the agroecosystems. For this reason, new management alternatives are being sought, such as the activation of systemic resistance. Induced by the application of salicylic acid that causes physical, chemical and molecular changes such as lignification or the induction of various proteins related to pathogenesis that does not allow the entry of the hyphae of pathogenic fungi, being a sustainable alternative.

Purpose: The purpose of this research was the evaluation of the effect of salicylic acid on the development of the symptoms of wheat leaf spot caused by Zymoseptoria tritici, and to establish its probable changes on the yield components in cultivars Biointa 3004 and Buck sy 200.

Methodology: The study was carried out at the Julio Hirschhorn experimental station of the National University of La Plata (UNLP), for which the cultivars Biointa 3004 and Buck sy 200 were used, the experimental design was in divided plots with three replications and was constituted by the following treatments: Control, inoculated with Zymoseptoria tritici, treated with salicylic acid and treated with salicylic acid and inoculated with Zymoseptoria tritici. and the tests were carried out in the winterspring season of 2012 and 2013 and the percentage of pycnidia, the percentage of necrotic area, the number of empty spikelets, the number of grains of the spike, and the weight of 1000 grains were determined. 
Results and conclusions: The pycnidia coverage decreased by $45 \%$; the necrotic area, by $40 \%$; in addition, the number of empty spikelets decreased by $4 \%$ and the number of grains per spike increased (5\%) and the weight of 1000 grains (2\%). It is shown that salicylic acid greatly controls the negative effects of the disease and stimulates the yield components, improving the productivity of two cultivars evaluated.

Keywords: Zymoseptoria tritici, Triticum aestivum, resistance induction, wheat leaf stain.

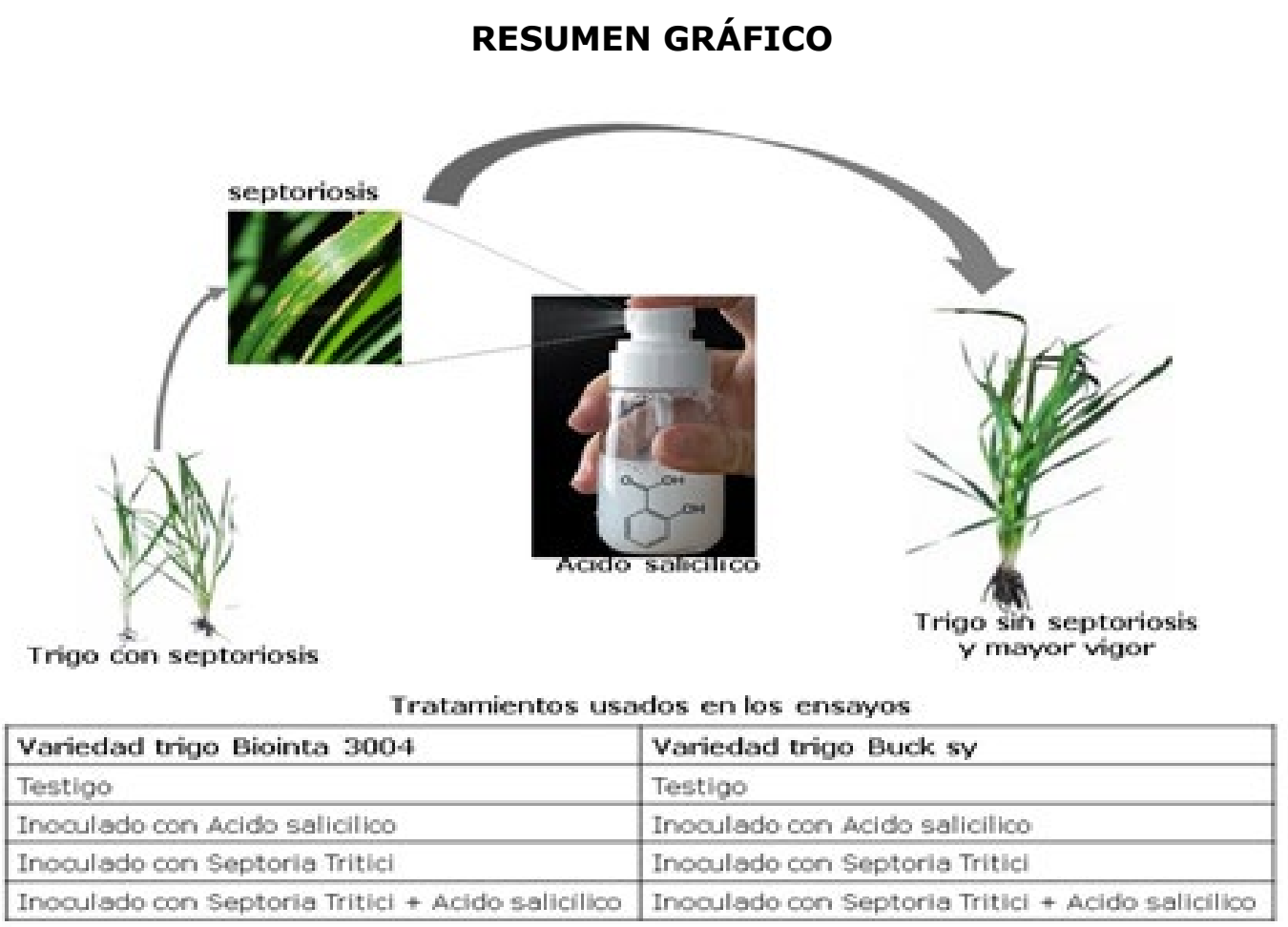

\section{INTRODUCCIÓN}

El trigo (Triticum aestivum) es el cereal de mayor distribución geográfica en el mundo y su demanda ha aumentado con el incremento de la población. Uno de los problemas en la producción de trigo es la mancha de hoja, o septoriosis, que es provocada por Zymoseptoria tritici (Z. tritici), en el estado anamorfo o asexual del hongo; mientras que el estado sexual o teleomorfo se conoce como Mycosphaerella graminícola (Stukenbrock et al., 2010). En la actualidad, la septoriosis del trigo está presente en las principales zonas de cultivo del mundo y está catalogada como una de las enfermedades de alto impacto en la producción del cultivo de trigo en Australia, Europa,
América del Norte y del Sur, con pérdidas hasta del $60 \%$ en el rendimiento (Testa et al., 2015; Gemechu et al., 2019). Por otra parte, se ha registrado que más del $70 \%$ de los fungicidas aplicados en trigo son para el control de la septoriosis, debido a que es una enfermedad endémica en las regiones del sudeste, áreas húmedas y sub-húmedas de Argentina. Esto causa pérdidas significativas en el rendimiento del cultivo, comparables con los daños ocasionados por la roya negra, anaranjada y la fusariosis de la espiga (Stocco, 2014).

Los síntomas de la enfermedad se caracterizan por pequeñas manchas foliares, cloróticas e 
irregulares usualmente en las hojas inferiores. Posteriormente, estas se expanden a las hojas superiores y su incremento en la severidad de $Z$. tritici en las hojas es causado por malas prácticas agronómicas como: implementación de monocultivo, manejo inadecuado de desechos de cosechas anteriores y mal manejo de las semillas, lo que ha causado mayores pérdidas en rendimiento y baja calidad del grano debido a la enfermedad (Goodwin, 2007; Velazquez, 2012).

El manejo de la enfermedad se da mediante el uso de umbrales de acción que justifiquen la aplicación de alguna técnica de control en particular, como son los fungicidas (Egan et al., 2020). Una alternativa no convencional es mediante la activación del sistema inmune; en particular, la resistencia sistémica adquirida (RSA) con la aplicación exógena de sustancias químicas, precursores de la cascada enzimática para la activación de las proteínas PR (proteínas relacionadas con la patogénesis en inglés pathogenesis-relared) que generan resistencia a fitopatógenos (Fu, 2013; Camarena, 2007).

La RSA se basa en el reconocimiento del invasor y un evento subsecuente de transducción de señales que conduce a la activación de las defensas. En muchos casos, la infección localizada induce resistencia a un amplio espectro de patógenos tales como hongos, bacterias o virus, esta resistencia se expresa localmente en el sitio de ataque por el patógeno y sistémicamente, en partes no infectadas de la planta (Vidhyasekaran, 2020). Así, la infección predispone a la planta a resistir efectivamente a ataques posteriores de patógenos.

Uno de los compuestos más estudiado para la activación RSA es el ácido salicílico (AS) que genera una serie de reacciones involucradas en la señalización de defensa de la planta (Wu et al., 2016; Vlot et al., 2008). También el AS se encuentra involucrado en procesos como germinación de semillas, crecimiento celular, respiración, cierre de estomas, expresión de genes asociados a senescencia, respuesta a estrés abiótico, termogénesis y resistencia a enfermedades (Vidhyasekaran, 2020).

Con base en lo anterior, el objetivo del trabajo fue evaluar el efecto del ácido salicilico sobre el avance de los síntomas de la Septoriosis del trigo en las primeras cuatro hojas expedidas; además, establecer su efecto sobre los componentes de rendimiento.

\section{MATERIALES Y MÉTODOS}

Los ensayos se llevaron a cabo en la Estación Experimental Julio Hirschhorn de la Facultad de Ciencias Agrarias y Forestales de la Universidad Nacional de la Plata, el Instituto de Fisiología Vegetal (INFIVE) y Centro de Investigaciones en Fitopatologías (CIDEFI), en la localidad de la Plata, de la Provincia de Buenos Aires (Argentina), en las campañas de invierno-primavera de los años 20122013 , con temperatura media de 14,2 y 15,2 ${ }^{\circ} \mathrm{C}$ respectivamente. Se utilizaron las variedades de trigo Buck sy 200 con resistencia moderada $Z$. tritici, buena calidad industrial, de ciclo intermedio y alto potencial de rendimiento; mientras que la variedad Biointa 3004, presenta susceptibilidad a Z. tritici, un ciclo de vida largo, resistencia al vuelco, tolerancia al desgrane y su calidad industrial es intermedia. Cada variedad presentó 4 tratamientos: Control (C) sin aplicación de productos químicos, tratado con ácido salicílico (AS), inoculado con Z. tritici (Sep) y tratado con ácido salicílico e inoculado con $Z$. tritici (AS+Sep). Se sembraron las semillas en parcelas de $4 \mathrm{~m}^{2}(2 \times 2)$, con densidad de siembra de 250 plantas $/ \mathrm{m}^{2}$ y fertilizadas preemergencias con $70 \mathrm{~kg} / \mathrm{ha}$ de fosfato diamónico y 40 
$\mathrm{kg} / \mathrm{ha}$ de urea. El diseño experimental que se utilizó para los ensayos fue bloques completos al azar con tres repeticiones.

\section{Preparación y aplicación del ácido salicílico}

Se prepararon soluciones del AS en concentración $100 \mu \mathrm{M}$ siguiendo el protocolo descrito por Tejada et. al (1998). La aplicación se efectuó en el momento que aparecieron dos hojas expandidas en la planta y se asperjaron los tratamientos AS y AS+Sep hasta punto de goteo; para realizar esta labor se utilizó una bomba de aspersión manual. Los tratamientos C y Sep fueron cubiertos por 2 horas con bolsas de polietileno para evitar la contaminación de los tratamientos por deriva.

\section{Preparación del inoculo de $Z$. tritici e ino- culación en campo}

La cepa de $Z$. tritici fue aislada de muestras de hojas de trigo de la localidad de 9 de Julio de la provincia de Buenos Aires-Argentina (FALP9J008) y utilizada por su capacidad de virulencia y patogenicidad (Stocco, 2014). La cepa se multiplicó en cajas de Petri que contenían medio Agar-Malta (30 g/L de extracto de malta, $5 \mathrm{~g} / \mathrm{L}$ de peptona, $2 \mathrm{~g} / \mathrm{L}$ de extracto de levadura y 20 $\mathrm{g} / \mathrm{L}$ de agar), y se incubó a $28^{\circ} \mathrm{C}$ por 7 días. $\mathrm{A}$ las cajas de Petri se les adicionó agua estéril y se procedió a raspar la superficie para extraer las esporas. Se filtró la suspensión resultante, con el fin de eliminar restos del medio de cultivo, y se llevó a una concentración de $1 \times 10^{6}$ esporas/ $\mathrm{mL}$. Se hizo necesario añadir Tween 20 a una concentración de $0,05 \%(\mathrm{v} / \mathrm{v})$, para una mejor adherencia a la superficie de las hojas de trigo.

Para los tratamientos AS+Sep y Sep, el inoculo de $Z$. tritici fue asperjado en horarios de la tarde hasta punto de goteo; además, se aplicó riego en intervalos de tres horas por un periodo de 72 horas, con el propósito de mantener la humedad relativa mayor al $80 \%, y$, de este modo, evitar la deshidratación de las esporas del hongo, asegurando una buena infección del patógeno. Los tratamientos control y AS fueron cubiertos con polietileno para evitar contaminación por deriva.

\section{Cuantificación de los síntomas de mancha de la hoja}

La evaluación de la enfermedad se realizó a 50 plantas por unidad experimental en los dos cultivares, a los 25 días postinoculación con $Z$. tritici, mediante la escala de Rosielle, que cuantifica el porcentaje de área necrosada (AN) y porcentaje de cobertura picnidial (Cob-P); se determinó en las 4 primeras hojas expandidas (Ramírez et al., 2016).

\section{Cuantificación de los componentes del rendimiento}

Las plantas se cosecharon y llevaron al laboratorio, donde se determinó los componentes del rendimiento: número de espiguillas por espiga; número de espiguillas vacías; número de granos por espiga; y peso de 1000 granos del macollo principal.

\section{Análisis estadístico}

Los datos obtenidos de las diferentes determinaciones (cuantificación de la enfermedad y componentes de rendimiento) se sometieron a un análisis de varianza (ANOVA) de un solo factor (tratamiento) mediante el software Infostat (Rienzo et al., 2014); sus medias se contrastaron con el test de Fisher utilizando una significancia del $5 \%(P \leq 0,05)$. Además, se aplicó la prueba Shapiro-Wilks para determinar que los datos tomados en campo presentan una distribución normal.

\section{RESULTADOS Y DISCUSIÓN \\ Evaluación de la aplicación exógena de AS en el control de Z. tritici}

La evaluación en el año 2012 del cultivar Biointa 3004 se muestra en la Tabla 1. Los efectos de la enfermedad fueron más notorios en 
la primera hoja, respecto a las más jóvenes, pasando del $98,3 \%$ A-N en la primera hoja, al $5 \%$ en la cuarta hoja. El A-N de la primera hoja se redujo con la aplicación de AS, incluso con valores inferiores al C (del 15 al 9\%). De igual forma, se observó una reducción del 70,7\% en el tratamiento AS+Sep. Con respecto a Cob$\mathrm{P}$, este presentó una disminución del $30 \%$ en la primera hoja a $0 \%$ en la cuarta hoja en el tratamiento AS+Sep. Respecto a los AS y el C, estos no presentaron diferencias significativas.

En la segunda hoja, el AS no mostró diferencias significativas respecto al $C$ en $A-N$; $\sin$ embargo, la aplicación de AS manifestó reducción del 58,3\% y Cob-P disminuyó un $41 \%$. En la tercera hoja el AS redujo el A-N en un $34 \%$ y Cob-P un $47 \%$. Tanto en el $C$ como el tratamiento con AS no se observaron cambios en estos parámetros.

En la cuarta hoja del tratamiento AS+Sep, se observó una reducción del 3,7\% y 3,5 \% en $\mathrm{A}-\mathrm{N}$ y Cob-P respectivamente. Para el año 2013, las tendencias fueron similares en cuanto a la reducción de A-N y Cob-P por la aplicación de AS en el tratamiento que fue infectado con Z. tritici.

Tabla 1. Evaluación del control de Z. tritici con la aplicación de AS en cultivar Biointa 3004.

\begin{tabular}{lccccc} 
Biointa 3004 & \multicolumn{2}{c}{ A-N (\%) } & \multicolumn{2}{c}{ Cob-P (\%) } \\
\hline Tratamientos & $\mathbf{N}^{\circ}$ de hojas & Año 2012 & Año 2013 & Año 2012 & Año 2013 \\
C & 1 & $15,3^{\mathrm{b}}$ & $11,2^{\mathrm{a}}$ & $2,0^{\mathrm{a}}$ & $9,5^{\mathrm{a}}$ \\
\hline Sep & 1 & $98,3^{\mathrm{c}}$ & $99,3^{\mathrm{c}}$ & $90,7^{\mathrm{b}}$ & $96,1^{\mathrm{c}}$ \\
\hline AS & 1 & $9,3^{\mathrm{a}}$ & $4,0^{\mathrm{a}}$ & $1,7^{\mathrm{a}}$ & $5,0^{\mathrm{a}}$ \\
\hline AS+Sep & 1 & $70,7^{\mathrm{a}}$ & $75,8^{\mathrm{b}}$ & $61,0^{\mathrm{c}}$ & $69,1^{\mathrm{b}}$ \\
\hline C & 2 & $2,4^{\mathrm{a}}$ & $2,2^{\mathrm{a}}$ & $0,5^{\mathrm{a}}$ & $0,3^{\mathrm{a}}$ \\
\hline Sep & 2 & $99,3^{\mathrm{c}}$ & $99,3^{\mathrm{c}}$ & $81,0^{\mathrm{b}}$ & $79,0^{\mathrm{c}}$ \\
\hline AS & 2 & $1,1^{\mathrm{a}}$ & $1,1^{\mathrm{a}}$ & $0,5^{\mathrm{a}}$ & $0,3^{\mathrm{a}}$ \\
\hline AS+Sep & 2 & $41,0^{\mathrm{c}}$ & $38,0^{\mathrm{b}}$ & $40,0^{\mathrm{c}}$ & $26,7^{\mathrm{d}}$ \\
\hline C & 3 & $0,3^{\mathrm{a}}$ & $0,3^{\mathrm{a}}$ & $0,5^{\mathrm{a}}$ & $0,2^{\mathrm{a}}$ \\
\hline Sep & 3 & $45,3^{\mathrm{b}}$ & $32,7^{\mathrm{a}}$ & $49,0^{\mathrm{b}}$ & $22,1^{\mathrm{b}}$ \\
\hline AS & 3 & $0,2^{\mathrm{a}}$ & $0,1^{\mathrm{a}}$ & $0,2^{\mathrm{a}}$ \\
\hline AS+Sep & 3 & $6,0^{\mathrm{c}}$ & $8,2^{\mathrm{a}}$ & $1,0^{\mathrm{a}}$ & $0,7^{\mathrm{a}}$ \\
\hline C & 3 & $0,4^{\mathrm{a}}$ & $0,2^{\mathrm{a}}$ & $0,5^{\mathrm{a}}$ & $0,1^{\mathrm{a}}$ \\
\hline Sep & 4 & $4,7^{\mathrm{b}}$ & $7,9^{\mathrm{b}}$ & $3,5^{\mathrm{b}}$ & $3,2^{\mathrm{b}}$ \\
\hline AS & 4 & $0,0^{\mathrm{a}}$ & $0,2^{\mathrm{a}}$ & $0,0^{\mathrm{a}}$ & $0,1^{\mathrm{a}}$ \\
\hline AS+Sep & $1,0^{\mathrm{a}}$ & $1,0^{\mathrm{a}}$ & $0,0^{\mathrm{a}}$ & $0,2^{\mathrm{a}}$ \\
\hline
\end{tabular}

ANOVA para cada hoja, aplicando el test LSD Fisher $(P \leq 0,05)$ entre las medias. Letras diferentes por columna indican diferencias significativas. A-N área necrosada, Cob-P cobertura de picnidios, C tratamiento Control, Sep tratamiento infectado con Z. tritici, AS tratado con ácido salicílico y AS+Sep tratado con ácido salicílico e infectado con Z. tritici.

Fuente: Autores. 
La evaluación del año 2012 del cultivar Buck sy 200 se muestra en la Tabla 2. Los efectos de la enfermedad son más notorios en la primera hoja que en las hojas más jóvenes, disminuyendo de un $10 \%$ del $\mathrm{A}-\mathrm{N}$ en la primera hoja al $0 \%$ en la cuarta. El tratamiento con AS tiende a disminuir el A-N y Cob-P pero no difiere significativamente respecto a $C$. En la segunda hoja el tratamiento con AS y $C$ no tienen cambios significativos en el A-N y Cob$P$, mientras que en el tratamiento $A S+$ Sep se observó disminución del $58,7 \%$ en el $\mathrm{A}-\mathrm{N}$ y la Cob-P un $62 \%$.

En cuanto a la tercera hoja, se redujo el A-N y Cob-P en $19 \%$ y $20 \%$ respectivamente. El C y el tratamiento con AS no mostraron síntomas ni signos de la enfermedad para la cuarta hoja. Para el año 2013 (Tabla 2), las tendencias fueron similares a las del año 2012 con un incremento moderado de la enfermedad.

Tabla 2. Evaluación del control de Z. tritici con la aplicación de AS en cultivar Bluck sy 200.

\begin{tabular}{|c|c|c|c|c|c|}
\hline \multirow{2}{*}{$\begin{array}{l}\text { Buck sy } 200 \\
\text { Tratamientos }\end{array}$} & \multicolumn{2}{|c|}{ A-N (\%) } & \multicolumn{2}{|c|}{ Cob-P (\%) } & \multirow[b]{2}{*}{ año 2013} \\
\hline & $N^{\circ}$ de hojas & año 2012 & año 2013 & año 2012 & \\
\hline C & 1 & $4,9^{a}$ & $1,8^{\mathrm{a}}$ & $1,0^{\mathrm{a}}$ & $0,3^{\mathrm{a}}$ \\
\hline Sep & 1 & $99,3^{c}$ & $90,3^{c}$ & $81,0^{c}$ & $90,2^{c}$ \\
\hline AS & 1 & $5,0^{a}$ & $2,7^{a}$ & $0,3^{\mathrm{a}}$ & $0,3^{\mathrm{a}}$ \\
\hline AS+Sep & 1 & $89,3^{b}$ & $70,6^{b}$ & $42,7^{b}$ & $67,8^{b}$ \\
\hline C & 2 & $0,3^{a}$ & $0,3^{a}$ & $0,3^{\mathrm{a}}$ & $0,3^{\mathrm{a}}$ \\
\hline Sep & 2 & $95,1^{c}$ & $98,3^{c}$ & $79,7^{c}$ & $90,2^{c}$ \\
\hline AS & 2 & $1,5^{\mathrm{a}}$ & $0,2^{a}$ & $0,3^{a}$ & $0,1^{\mathrm{a}}$ \\
\hline AS+Sep & 2 & $36,4^{b}$ & $68,9^{b}$ & $17,3^{a}$ & $47,8^{b}$ \\
\hline $\mathrm{C}$ & 3 & $0,0^{c}$ & $0,0^{\mathrm{a}}$ & $0,0^{a}$ & $0,0^{\mathrm{a}}$ \\
\hline Sep & 3 & $19,3^{b}$ & $29,7^{b}$ & $20,0^{b}$ & $18,3^{b}$ \\
\hline AS & 3 & $0,0^{a}$ & $0,0^{a}$ & $0,0^{\mathrm{a}}$ & $0,0^{\mathrm{a}}$ \\
\hline AS+Sep & 3 & $0,0^{a}$ & $0,0^{a}$ & $0,0^{\mathrm{a}}$ & $0,0^{\mathrm{a}}$ \\
\hline $\mathrm{C}$ & 4 & $0,0^{a}$ & $0,0^{\mathrm{a}}$ & $0,0^{a}$ & $0,1^{\mathrm{a}}$ \\
\hline Sep & 4 & $0,0^{\mathrm{a}}$ & $7,9^{\mathrm{b}}$ & $0,0^{a}$ & $3,2^{\mathrm{b}}$ \\
\hline AS & 4 & $0,0^{a}$ & $0,0^{\mathrm{a}}$ & $0,0^{a}$ & $0,0^{\mathrm{a}}$ \\
\hline AS+Sep & 4 & $0,0^{a}$ & $0,0^{a}$ & $0,0^{a}$ & $0,0^{\mathrm{a}}$ \\
\hline
\end{tabular}

ANOVA para cada hoja, aplicando el test LSD Fisher $(P \leq 0,05)$ entre las medias. Las letras diferentes por columna indican diferencias significativas. A-N área necrosada, Cob-P cobertura de picnidios, C Control, Sep infectado con $Z$. tritici, AS tratado con ácido salicílico y AS + Sep o tratado con ácido salicílico e infectado con Z. tritici.

Fuente: Autores 
A partir de los experimentos en campo de los cultivares comerciales Biointa 3004 y Buck sy 200 se puede deducir que el AS tiene un efecto positivo sobre el control del patógeno Z. tritici, este efecto podría estar relacionado con los mecanismos de señalización en la repuesta de defensa a patógenos (Wu et al., 2016). El AS es un factor de transcripción del gen NPR1 que activa la transducción de defensas y los mecanismos que tiene la planta para evitar la infección patogénica en los cultivares evaluados (Fu, 2013; Moreauet et al., 2012; Fu et al., 2012). Por otra parte, Wang et al., (2018) demostró que la aplicación de AS en plantas de trigo disminuye un $40 \%$ la infección por Phaeosphaeria nodorum, esta disminución se debe a que las hifas del hongo no pueden penetrar las células de la epidermis y las células de la vaina por la lignificación prematura, acumulación de calosa y concentración de calcio en la pared de las células producto de la activación del sistema de defensa. Otros reportes indican que en plantas de trigo los niveles de AS, calosa, compuestos fenólicos, peroxidasa, fenilalanina amonio liasa y polifenol oxidasa aumentan la resistencia a Fusarium graminearum (Sorahinobar et al., 2016), por lo que se puede inferir que la aplicación exógena del AS puede controlar diferentes fitopatógenos mediante la inducción de la resistencia sistémica adquirida.

\section{Determinación de los componentes de rendimientos \\ Número de espiguillas}

El número de espiguillas por espiga no presentó cambios significativos entre los tratamientos para los dos años de evaluación, quedando determinado en 21 espiguillas en los dos cultivares. Esto se debe a la gran estabilidad genética en este carácter, indicando que AS y la enfermedad no afectan la activación de los genes encargados de la formación y determinación del número de espiguillas por espiga (Tabla 3 y Tabla 4). Las evidencias bibliográficas indican que el AS y Z. tritici no afectan la determinación del número de espiguillas, pero sí afectan en número de flores y fecundación de las mismas (Rodríguez et al., 2008; Wada et al., 2010; Appu, 2014; Martín et al., 2015)

\section{Número de espiguillas vacías}

En el cultivar Biointa 3004 en el año 2012, se observó un aumento del $16 \%$ en el número de espiguillas vacías. En las plantas que fueron infectadas con Z. tritici, para el año 2013 el daño fue mayor, aumentando al 29\%. Los tratamientos con AS y AS+Sep redujeron el número de espiguillas vacías en un $66 \%$ en promedio, para el cultivar Buck sy 200. En el año 2012 - 2013 se observó un aumento del 21,8 $\%$ y $36,6 \%$ en el tratamiento con Sep respectivamente, mientras que los tratamientos con AS y AS+Sep no modifican este parámetro comparado con el control (Tabla 3).

El tratamiento infectado con $Z$. tritici se afecta de forma negativa aumentando el número de espiguillas vacías entre el $16 \%$ y el $30 \%$, mientras que la aplicación de AS muestra un efecto positivo sobre este componente evitando la pérdida de granos $y$, en consecuencia, un menor número de espiguillas vacías. Estos resultados son similares a los reportados por Seadh y Metwally (2015) quienes demostraron que la aplicación exógena de AS mejora el rendimiento y la productividad al controlar los efectos negativos provocados por los patógenos Cephalosporium sp., Fusarium culmorum, F. moniliforme y $F$. graminearum sobre los componentes del rendimiento en cultivares de trigo egipcios. 
Tabla 3. Variables evaluadas de componentes de rendimiento del cultivar Biointa 3004 y tratadas con AS e inoculadas con Z. tritici en campo para los años 2012 y 2013

\begin{tabular}{l|c|c|c|c|c|c|c|c|}
\hline & \multicolumn{3}{c}{ C } & \multicolumn{2}{c}{ Sep } & \multicolumn{1}{c}{ AS } & AS+Sep \\
\cline { 2 - 8 } Biointa 3004 & Año & Año & Año & Año & Año & Año & Año & Año \\
& $\mathbf{2 0 1 2}$ & $\mathbf{2 0 1 3}$ & $\mathbf{2 0 1 2}$ & $\mathbf{2 0 1 3}$ & $\mathbf{2 0 1 2}$ & $\mathbf{2 0 1 3}$ & $\mathbf{2 0 1 2}$ & $\mathbf{2 0 1 3}$ \\
\hline$N^{\circ}$ de espiguillas & $21,0^{\mathrm{a}}$ & $21,0^{\mathrm{A}}$ & $21,0^{\mathrm{a}}$ & $21,0^{\mathrm{A}}$ & $21,0^{\mathrm{a}}$ & $21,0^{\mathrm{A}}$ & $21,0^{\mathrm{a}}$ & $21,0^{\mathrm{A}}$ \\
\hline$N^{\circ}$ de espiguillas vacías & $2,7^{\mathrm{a}}$ & $3,0^{\mathrm{B}}$ & $3,1^{\mathrm{b}}$ & $4,1^{\mathrm{C}}$ & $2,7^{\mathrm{a}}$ & $2,1^{\mathrm{A}}$ & $2,6^{\mathrm{a}}$ & $2,2^{\mathrm{A}}$ \\
\hline$N^{\circ}$ de granos por espiga & $49,0^{\mathrm{b}}$ & $48,5^{\mathrm{B}}$ & $45,1^{\mathrm{a}}$ & $46,2^{\mathrm{A}}$ & $49,9^{\mathrm{b}}$ & $51,2^{\mathrm{C}}$ & $49,1^{\mathrm{b}}$ & $49,2^{\mathrm{B}}$ \\
\hline Peso de 1000 granos $(\mathrm{g})$ & $27,0^{\mathrm{b}}$ & $28,3^{\mathrm{B}}$ & $25,4^{\mathrm{a}}$ & $25,9^{\mathrm{A}}$ & $29,1^{\mathrm{C}}$ & $30,3^{\mathrm{C}}$ & $27,6^{\mathrm{b}}$ & $28,2^{\mathrm{B}}$ \\
\hline
\end{tabular}

Control (C), Sep (infectado con Z. tritici), AS (tratado con ácidos salicílico) y AS+Sep (tratado con ácido salicílico e infectado con Z. tritici). ANOVA para cada variable, test LSD Fisher $(P \leq 0,05)$ entre las medias de los tratamientos. Las Letras diferentes horizontales por componentes de rendimiento indican diferencias significativas, letras con minúsculas año 2012 y letras en mayúsculas año 2013.

Fuente: Autores.

\section{Número de granos de la espiga principal}

Para el año 2012, el cultivar Biointa 3004 presentó una disminución del 6,9\% en el número granos por espiga en plantas inoculadas con Sep, mientras que los tratamientos AS y AS+Sep no mostraron cambios significativitos en este componente respecto al control. En el año 2013, en el tratamiento con Sep presentó disminución en un $4,7 \%$, mientras que las plantas tratadas con AS mostraron un aumento del 5,6\% (Tabla 3).

Los resultados del cultivar Buck sy 200 fueron similares al cultivar Biointa 3004, para los dos años de evaluación a excepción de tratamiento con AS+Sep que aumentó el número de granos por espiga en un 2,9\% (Tabla 4). Muthukrishnan et al., (2014) y Seadh et al., (2015) reportan que la aplicación exógena de AS en cultivares de trigo mejoró el número de granos por espiga en el orden de $2,1 \%$ a $7,3 \%$, sin causar efectos negativos sobre la calidad industrial del grano, modificando de forma positiva el número de espiguillas vacías por un aumento en la emisión de flores y una mayor fecundación de las mismas. Cabe aclarar que, en nuestros ensayos, no se realizó evaluación en la calidad industrial de los granos.

\section{Peso de 1000 granos de la espiga principal}

La evaluación realizada en los dos años muestra aumento en el peso de los granos en las plantas tratadas con AS (4,4\% año 2012 y $7,6 \%$ año 2013), mientras que el tratamiento con AS+Sep no tuvo diferencias significativas respecto a C. Por otra parte, el tratamiento inoculado con Sep mostró menores valores en el peso de los granos 1000 granos, con valores de $5,9 \%$ en el año 2012 y $-8,4 \%$ en el año 2013 (Tabla 3).

El cultivar Buck sy 200 presentó un aumento en el peso de 1000 granos respecto al cultivar Biointa 3004, mientras que en las plantas tratadas con AS aumentó un $9,1 \%$ y un $4,4 \%$ en el 2012 y 2013 respectivamente (Tabla 4). Se observaron resultados similares en el tratamiento con AS + Sep aumentados un 5,5\% y un $1,5 \%$ respectivamente. Con respecto a las plantas infectadas con Sep y C no presentaron cambios significativos en el peso de los 1000 granos. 
Tabla 4. Variables evaluadas de componentes de rendimiento del cultivar Buck sy 200 y tratadas con AS e inoculadas con Z. tritici en campo para los años 2012 y 2013.

\begin{tabular}{|c|c|c|c|c|c|c|c|c|}
\hline \multirow[b]{2}{*}{ Buck sy 200} & \multicolumn{2}{|c|}{ C } & \multicolumn{2}{|c|}{ Sep } & \multicolumn{2}{|c|}{ AS } & \multicolumn{2}{|c|}{ AS+Sep } \\
\hline & $\begin{array}{c}\text { Año } \\
2012\end{array}$ & $\begin{array}{c}\text { Año } \\
2013\end{array}$ & $\begin{array}{c}\text { Año } \\
2012\end{array}$ & $\begin{array}{c}\text { Año } \\
2013\end{array}$ & $\begin{array}{c}\text { Año } \\
2012\end{array}$ & $\begin{array}{c}\text { Año } \\
2013\end{array}$ & $\begin{array}{c}\text { Año } \\
2012\end{array}$ & $\begin{array}{c}\text { Año } \\
2013\end{array}$ \\
\hline Número de espiguillas & $21,0^{a}$ & $21,0^{A}$ & $21,0^{\text {a }}$ & $21,0^{A}$ & $21,0^{a}$ & $21,0^{\mathrm{A}}$ & $21,0^{\text {a }}$ & $21,0^{A}$ \\
\hline Número espiguillas vacías & $3,2^{a}$ & $3,0^{A}$ & $3,9^{b}$ & $4,1^{\mathrm{B}}$ & $3,2^{a}$ & $3,0^{A}$ & $3,1^{\text {a }}$ & $3,0^{A}$ \\
\hline Número de granos por espiga & $47,8^{b}$ & $48,5^{\mathrm{B}}$ & $46,2^{a}$ & $45,6^{A}$ & $48,3^{b}$ & $48,1^{\mathrm{B}}$ & $47,0^{b}$ & $49,9^{c}$ \\
\hline Peso de 1000 granos (g) & $33,3^{\mathrm{b}}$ & $33,3^{\mathrm{B}}$ & $32,1^{\mathrm{a}}$ & $31,7^{A}$ & $35,3^{d}$ & $35,3^{D}$ & $34,3^{c}$ & $34,3^{c}$ \\
\hline
\end{tabular}

C (control), Sep (tratamiento con inoculadas con Z. tritici), AS (tratamiento asperjado con ácido salicílico) y AS+Sep (tratamiento asperjado con ácido salicílico e inoculado con Z. tritici). ANOVA para cada variable, aplicando el test LSD Fisher $(P \leq 0,05)$ entre las medias de los tratamientos. Las Letras diferentes horizontales, por componentes de rendimiento, indican diferencias significativas, letras con minúsculas año 2012 y letras en mayúsculas año 2013.

Fuente: Autores

La septoriosis influye en la calidad del grano por presentar una disminución significativa en el peso de 1000 granos. Estos datos son similares a los reportados en los estudios de Rodríguez et al., (2008), en los que se presentaron pérdidas en el peso de 1000 granos debido a la presencia del patógeno $Z$. tritici, afectando directamente el rendimiento del cultivo. En los tratamientos que se aplicó AS, para los dos cultivares evaluados, se observó un aumento en el peso de los granos del $10 \%$ y $6 \%$. estos datos son congruentes con las investigaciones hechas por Martínez et al., (2013) y Sneadh et al., (2015), quienes observaron que la aplicación de AS a las plantas de trigo, mejoró el peso de los granos entre el 4,6 y el $17,0 \%$ y el rendimiento por hectárea aumenta entre $600-800 \mathrm{Kg}$. Este efecto también fue observado en otros cultivos, como papaya y chile habanero (Guzmán et al., 2012; Martín et al., 2015). En este estudio, la inoculación con $A S+Z$. tritici controló el efecto provocado por la enfermedad en el peso de los granos. Es posible que esto se deba a la resistencia sistémica adquirida reconoce al invasor y posteriormente se da la transducción de señales que conduce a la activación de defensas impidiendo el daño de las hojas, lo que evita la pérdida de la actividad fotosintética y la senescencia prematura de la hoja (Sneadh et al., 2015).

\section{CONCLUSIONES}

La activación temprana de la resistencia sistémica adquirida mediada por la aplicación exógena de AS controló la cobertura de picnidios en un $45 \%$ y el área necrosada en un $40 \%$. Estos resultados estarían relacionados con el aumento de la concentración de lignina, calosa y calcio en la pared celular que evita la infección de $Z$. tritici en las hojas de trigo y disminuye los efectos adversos en los componentes de rendimiento. Además, en el tratamiento donde se aplicó AS, se estimula el incremento en algunos parámetros del rendimiento, lo que mejoraría los kilogramos de granos. Lo anterior abre una nueva perspectiva muy promisoria para el manejo sustentable de la enfermedad, debido a que el AS es un producto no contaminante para el medio ambiente y puede ser una alternativa ecológica dentro de una agricultura limpia, disminuyendo los impactos negativos en la fauna y la microfauna de las zonas trigueras en la república de Argentina. 


\section{CONTRIBUCIÓN DE LA AUTORÍA}

Primer autor (Efren Venancio Ramos Cabrera): metodología, investigación, análisis de datos, conceptualización, escritura - borrador original. Segundo autor (Zuly Yuliana Delgado Espinosa): escritura - revisión y edición. Tercer autor (Daniel Oscar Gimenez): logística, revisión, edición y análisis de datos, revisión.

\section{AGRADECIMIENTOS}

Los autores de este proyecto manifiestan sus agradecimientos a la universidad Nacional de la Plata - Argentina por facilitar el acceso a las instalaciones y toma de datos.

\section{LITERATURA CITADA}

Appu, M., y Muthukrishnan, S. (2014). Foliar Application of Salicylic Acid Stimulates Flowering and Induce Defense Related Proteins in Finger Millet Plants. Universal Journal of Plant Science 2(1): 14-18. https:// doi:10.18805/Ir.v0iOF.11302

Camarena, G., y De la Torre, R. (2007). Resistencia sistémica adquirida en plantas: estado actual. Revista Chapingo Serie Ciencias Forestales y Del Ambiente 13(2): 157-162.

Egan, P. A., Dicks, L. V., Hokkanen, H. M., y Stenberg, J. A. (2020). Delivering Integrated Pest and Pollinator Management (IPPM). Trends in Plant Science 25 (6): 577-589. https://doi. org/10.1016/j.tplants.2020.01.006

Fu, Z. Q., S. Yan, A. Saleh, W. Wang, J. Ruble, N. Oka, X. Dong. (2012). NPR3 and NPR4 are receptors for the immune signal salicylic acid in plants. Nature 486(7402): 228-232.

Fu, Z. y Xinnian, D. (2013). "Resistencia adquirida sistémica: convertir la infección local en defensa global". Revisión anual de biología vegetal 64 (1): 839-63. https://doi.org/10.1146/annurevarplant-042811-105606

Gemechu, B., Besufekad, A., y Mekuriaw, A. (2019). "Performance Evaluation of Improved Bread Wheat (Triticum Aestivum L.) Varieties and Production Technologies in Central High Lands of Ethiopia." African Journal of Agricultural Research 14 (7): 439-46. https:// doi:10.5897/ AJAR2018.13171
Goodwin, S. B. (2007). Back to basics and beyond: Increasing the level of resistance to Septoria tritici blotch in wheat. Australasian Plant Pathology 36(6): 532-538.

Guzmán, A., Borges, L., Pinzón, E., Ruiz y J. Zúñiga-Aguilar. (2012). Efecto del ácido salicílico y la nutrición mineral sobre la calidad de plántulas de chile habanero. Agronomía Mesoamericana, 23(2): 247-257. https://doi: 10.15517/ am.v23i2.6485

Martín, R., Nexticapan, A., y Larqué, A. (2013). Potential benefits of salicylic acid in food production. In salicylic acid (pp. 299-313). Springer, Dordrecht. https://DOI: 10.1007/978-94-007-6428-6_13

Martín, R., Nexticapan, A., Villanueva, V., Uicab, S., y Larque, A. (2015). Salicylic acid stimulates flowering in micropopagated gloxinia plants. Revista fitotecnia mexicana 38(2): 115-118.

Moreau, M., Tian, M., y Klessig, D. (2012). Salicylic acid binds NPR3 and NPR4 to regulate NPR1-dependent defense responses. Cell Research 22(12): 1631-1633. https:// doi:10.1038/ cr. 2012.100

Ramírez, J., Santa Rosa, R., Villaseñor, H., López, E., Martínez, E., y Espitia, E. (2016). Evaluation of varieties and even lines of bread wheat temporal in Valles Altos. Revista Mexicana de Ciencias Agrícolas, 7(3), 655-667.

Rienzo, J., Casanoves, F., Balzarini, M., Gonzalez, L., Tablada, M., y Robledo, C. (2014). InfoStat 2014. Grupo InfoStat, FCA. Universidad Nacional de Córdoba, Córdoba, ARG.

Rodríguez, M., Villaseñor, H., Leyva,G, Huerta, J., Huerta, J. y De los Santos, H. (2008). Efecto de Septoria tritici en el rendimiento de trigo de temporal en ambientes lluviosos de los valles altos centrales de México. Agrociencia 42(4): 435-442.

Seadh, S. E., y El-Metwally, M. (2015). Influence of antioxidants on wheat productivity, quality and seed-borne fungi management under NPK fertilization levels. Asian Journal of Crop Science 7(2): 87. https://doi: 10.21608/JPP.2017.37815

Sorahinobar, M., Niknam, V., Ebrahimzadeh, H., Soltanloo, H., Behmanesh, M., y Enferadi, S. T. (2016). Central Role of Salicylic Acid in Resistance of Wheat Against Fusarium graminearum. Journal of Plant Growth Regulation 35(2): 477-491. https://doi.org/10.1007/s00344-015-9554-1 
Stocco, M. C. (2014). Control biológico de Mycosphaerella graminicola, patógeno del trigo, con cepas de Trichoderma harzianum caracterizadas por su morfología, fisiología, actividad enzimática y molecular. Universidad Nacional de La Plata, La plata. http://sedici.unlp.edu.ar/handle/10915/42904

Stukenbrock, E., Jørgensen, F., Zala, M., Hansen, T., McDonald, B., y Schierup, M. (2010). Wholegenome and chromosome evolution associated with host adaptation and speciation of the wheat pathogen mycosphaerella graminicola. PLoS Genetics 6(12): e1001189. https://doi.org/10.1371/ journal.pgen.1001189

Testa, A., Oliver, R., y Hane, J. (2015). "Overview of Genomic and Bioinformatic Resources for Zymoseptoria Tritici." Experimental Mycology 79: 1316. https://doi.org/10.1016/j.fgb.2015.04.011

Velazquez, P. D., y Formento, A. N. (2012). Efecto de la fusariosis de la espiga (Fusarium graminearum y Fusarium sep.) sobre dos genotipos de trigo. XIV Jornadas Fitosanitarias Argentinas, 2331, F-63.

Vidhyasekaran, P. (2020). Bioengineering and Molecular Manipulation of Salicylic Acid Signaling
System to Activate Plant Immune Responses for Crop Disease Management. Springer, Dordrecht: 169-221. DOI: 10.1007/978-94-024-1940-5_5.

Vlot, A., Klessig, D., y Park, S. (2008). Systemic acquired resistance: the elusive signal(s). Current Opinion in Plant Biology 11(4): 436-442. https://doi.org/10.1016/j.pbi.2008.05.003

Wu, J., Sang, G., Kyu, Y., Ju, G., Sang, R., Ravi, G., Yong, H., Yiming, W., y Sun, T. (2016). Overexpression of a Pathogenesis-Related Protein 10 Enhances Biotic and Abiotic Stress Tolerance in Rice. The Plant Pathology Journal 32 (6): 55262. https://doi: 10.5423/PPJ.OA.06.2016.0141

Wada, K. C., Yamada, M., Shiraya, T., y Takeno, K. (2010). Salicylic acid and the flowering gene FLOWERING LOCUS T homolog are involved in poor-nutrition stress-induced flowering of Pharbitis nil. Journal of Plant Physiology 167(6): 447452. https://doi: 10.1016/j.jplph.2009.10.006

Wang, X., Wei, S., Jing, G., Xiu, M., Hai, W., y Da L. (2018). "Systemic Acquired Resistance, NPR1, and Pathogenesis-Related Genes in Wheat and Barley." Agricultural Sciences in China 17 (11): 2468-77. https://doi.org/10.1016/S20953119(17)61852-5
Conflicto de Intereses Los autores declaran no tener ningún conflicto de intereses 